\title{
Lateralization of Receptive Language Function Using near Infrared Spectroscopy
}

\author{
Natacha Paquette ${ }^{1,2}$, Berta Gonzalez-Frankenberger ${ }^{1,2}$, Phetsamone Vannasing ${ }^{1}$, Julie Tremblay ${ }^{1}$, \\ Olivia Florea $^{1,2}$, Renée Beland ${ }^{1,2}$, Franco Lepore ${ }^{1,2}$, Maryse Lassonde ${ }^{1,2}$ \\ ${ }^{1}$ Centre de Recherche, University Hospital Center Sainte-Justine, Montreal, QC, Canada; ${ }^{2}$ Centre de Recherche en Neuropsychologie \\ et Cognition, Université de Montréal, Montréal, QC, Canada \\ Email: maryse.lassonde@umontreal.ca
}

Received September $1^{\text {st }}, 2010$; revised September $8^{\text {th }}, 2010$; accepted September $13^{\text {th }}, 2010$

\begin{abstract}
In recent decades, functional magnetic resonance imaging ( $f M R I$ ) has proven to be more effective than the Wada test in the evaluation of language lateralization in special populations such as epileptic patients and children. However, fMRI requires that subjects remain motionless during data acquisition, making the assessment of receptive and expressive language difficult in young children and population with special needs. Near-Infrared spectroscopy (NIRS) is a noninvasive technique that has proven to be more tolerant to motion artifacts. The aim of the present study was to investigate the use of NIRS to assess receptive language patterns using a story listening paradigm. Four native French-speakers listened to stories read aloud by a bilingual speaker in both French and Arabic. To determine if the signal recorded was affected by episodic memory processes, a familiar story and an unknown story were presented. Results showed that listening to stories in French elicited a significantly higher left lateralized response than listening to stories in Arabic, independently of the familiarity of the story. These results confirm that NIRS is a useful non-invasive technique to assess receptive language in adults and can be used to investigate language lateralization among children and epileptic patients slated for epilepsy surgery.
\end{abstract}

Keywords: Language Lateralization, Near Infrared Spectroscopy (NIRS), Optical Imaging, Brain Mapping, Epilepsy

\section{Introduction}

Pre-surgical examination of epileptic patients traditionally includes language lateralization assessment [1]. However, language is a complex entity involving multiple levels of processing. Expressive language tasks (e.g. verbal fluency) are the most frequently used paradigms to investigate language lateralization [2,3]. Receptive language paradigms, however, may be more suitable when working with young children or patients with mental retardation as they do not require active participation of the subject [4]. A number of functional magnetic resonance imaging (fMRI) and positron emission tomography (PET) studies suggest a left temporo-frontal stream specifically involved during syntactic and semantic processing, both necessary to comprehension [5-7]. For instance, the processing of sentence with canonical Subject-Verb-Object structure (SVO) involves a pathway that includes the frontal inferior gyrus, the supplementary motor area and the temporal inferior gyrus [8]. These authors further suggest that the left temporal inferior gyrus would play a role in the integration of the Subject, the Verb and the Object into a semantically coherent sentence.

It thus appears that the integration of acoustic, semantic and syntactic processes is necessary for language comprehension [9]. Therefore, studies aiming to localize receptive language must rely on language tasks requiring integration of information processed in theses modules, such as coherent sentences or story comprehension tasks. Using fMRI, Schlosser, Aoyagi, Fulbright, Gore \& McCarthy (1998) observed a stronger activation of the left superior temporal sulcus compared to the right homologous region when English-speaking participants listened to English sentences [10]. In contrast, Turkish sentence presentation (control task) did not induce similar cerebral activations in any of the participants. Reporting similar results, Mazoyer et al. (1993) used fMRI and PET while participants were listening to Tamil (participants' unknown language) and French (participants' mother tongue) spoken stories. After subtraction, they found a stronger 
activation for French stories in the left superior and left medial temporal gyri [5].

Although these studies confirm a left hemisphere specialization for receptive language processing $[5,10]$, the specific involvement of different brain regions in each level of processing is still controversial [11]. Some authors suggest that other processes such as mental imagery and episodic memory retrieval may be implicated when subjects listen to stories [12]. For instance, activation that has been observed in the parieto-temporal junction and in the prefrontal medial cortex may be due to the latter processes $[11,13]$. Because mental imagery and episodic memory retrieval might be associated with story comprehension, their relation with receptive language processing must be further investigated. To our knowledge, no previous studies have investigated the impact of episodic memory and mental imagery processes on the brain activation patterns observed in receptive language processing. The use of a paradigm involving the presentation of a known story versus an unknown one might shed light on this matter.

The brain activation patterns of receptive language processing have been mainly studied with fMRI and PET. However, the invasive and restrictive nature of these techniques often make them inappropriate for young children, epileptic patients or patients with mental retardation $[2,14]$. To date, few studies have been conducted with near-infrared spectroscopy, a non-invasive technique that allows the measurement of blood oxygenation changes related to cerebral activation [15,16]. Also known as optical imaging, this technique is based on the light absorption properties of oxyhemoglobin ( $\mathrm{HbO}$ ) and deoxyhemoglobin (HbR) [17]. Good agreements have been observed between NIRS and fMRI, MEG and PET $[9,18,19]$ However, NIRS has a better temporal resolution than fMRI and its mobile and cheaper nature confers it with important advantages over the other techniques. Moreover, its configuration does not require subject containment, thus allowing the participant to move and speak. This makes it ideal while evaluating cognitive and linguistic functions or for research with young children and special populations $[2,4,9,20]$.

The present study aims to investigate the use of NIRS to assess brain lateralization patterns for receptive language functions in healthy adults while further controlling for episodic memory retrieval and mental imagery processes. Using a story listening task, we hypothesized that 1) French-speaking participants would show a greater left lateralized activation pattern in response to a French story compared to an Arabic one and, 2) if episodic memory retrieval affects the processing of the information, a different activation pattern should be observed in response to an unknown story (Martine and the Witch) compared to a known story (Snow White).

\section{Methodology}

\subsection{Participants}

Six healthy volunteers ( 3 men; mean age $=22$ years (16-28 years), SD $=3.85$ ) without neurological impairments were tested. The handedness of each subject was assessed using the Edinburgh Inventory [21]: four were right-handed and two were ambidextrous with a right predominance. All were native from the Quebec province, French-speaking and without knowledge of the Arabic language. Two subjects ( 1 man, 1 woman) were withdrawn from the analyses due to technical artifacts. This study has been approved by the Ethics Committee of the Ste-Justine University Hospital Center and informed consent was obtained from all participants.

\subsection{Optical Imaging Recording}

The data were gathered using a multi-channel spectrometer Imagent Tissue Oxymeter (ISS Inc., Champaign, III, USA) made of 50 sources emitting a $690 \mathrm{~nm}$ wavelength, 50 sources emitting a $830 \mathrm{~nm}$ wavelength and 16 detectors. The sources were placed on the subject's scalp using a rigid helmet adapted to the subject's head. Due to the anatomical variability of the subjects' head, two different helmet sizes were used $(57 \mathrm{~cm}$ and $59 \mathrm{~cm}$ ), and a standard montage was created for each of these helmets. Both montages were created using the software Brainsight TM Frameless 39 (Rogue Research, Canada) from a standardized MRI template, the Colin27 [22]. A total of 144 channels, using a source-detector distance from 2.9 $\mathrm{cm}$ to $6 \mathrm{~cm}$, were symmetrically laid out to cover the left cerebral regions known to be related to language functions (Broca's area and Wernicke's area), as well as the right homologous regions. Figure 1 shows the regions covered by the montage.

For each subject, the exact localization of each source and detector, as well as four fiducial points (nasion, left and right pre-auricular, and tip of the nose), were digitized and recorded using the stereotaxic system Brainsight to allow the individual reconstitution of the montage on the MRI template. Finally, a simultaneous EEG recording with four electrodes placed on the scalp (Fz, $\mathrm{Cz}, \mathrm{Pz}$ and $\mathrm{Oz}$ according to the $10 / 20$ international system) was carried out in order to control for the subjects' alert state during the task.

\subsection{Stimuli}

Two stories were used: Snow-White (known story) and Martine and the Witch (unknown story). Stories were recorded using Cool Edict ProTm in French and Arabic by the same speaker to control for acoustic and voice factors. The amplitude (between $64 \mathrm{~dB}$ and $76 \mathrm{~dB}$ ) and the duration of the stimuli (20 s each) were adjusted and stan- 

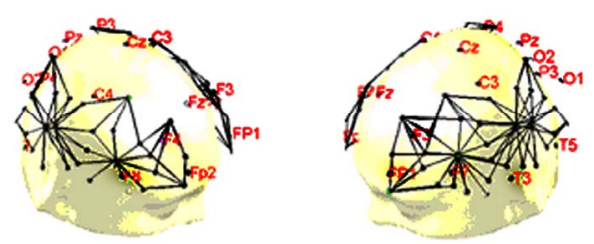

(a)
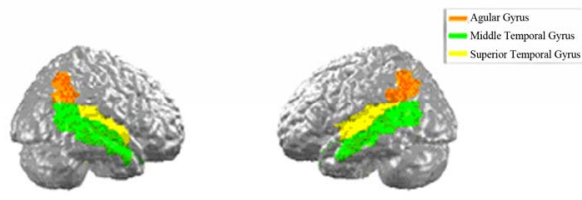

(b)

Figure 1. Cerebral regions covered by the standard montage (a) and cortical representation of the activated regions observed in all subjects (b).

dardized with the same software. All the stimuli were presented to the participants using MatLab 7.0.4 and transmitted by two loudspeakers located at an equal distance (approximately $155 \mathrm{~cm}$ ) from the subject, at ear level.

\subsection{Procedure}

Testing took place in a dark, sound-proof room. Subjects came twice to the laboratory. The experimental procedure and the language sequence were the same from one visit to the other. Participants were seated, with eyes closed. They were asked to relax in order to avoid abrupt movements and to listen carefully to the stories. They listened first to the story of Snow-White in both languages and, in a second testing session, to the story of Martine and the Witch in both languages. The time interval between both sessions was approximately two months to control for repeated measure reactivity. At each visit, participants listened to short extracts of the story read in French and the same story extracts read in Arabic. Each condition comprised 18 blocks with an interstimulus interval of 40s. Stories were presented in a continuous order to make it possible for the participant to follow the story. The order of the language was counter-balanced from one subject to the other (all the stimuli in French followed by all the stimuli in Arabic or all the stimuli in Arabic followed by all the stimuli in French). The total duration of each recording session was approximately $80 \mathrm{~min}$, including the set-up of the helmet, optodes, detectors and electrodes. At the end of each session, participants had to answer some questions related to the stories to further control for comprehen sion and attention levels as well as for the familiarity of the stories.

\subsection{Data Processing}

The data acquisition rate was $19.5312 \mathrm{~Hz}$ downsampled by a factor of 5 to lighten the data processing. The raw hemodynamic signal was normalized with a $10 \mathrm{~s}$ prestimulus time. Artefact rejection took place by withdrawing segments with light intensity amplitudes smaller than 100 DC or a normalized standard deviation higher than $50 \%$. The optical intensity of the raw data (DC) was filtered using a low frequency zero-phase digital filtering with a cut off frequency at $0.1 \mathrm{~Hz}$. A Modified Beer Lambert Law with a differential path length factor (DPF) correction according to the age of each subject was applied $[23,24]$. Finally, the variation in the $\mathrm{HbO}$ and $\mathrm{HbR}$ concentrations were averaged for each channel and each condition. Averages were co-registered and projected on the Colin27 standard MRI template [22] to visualize the activated brain regions. Subsequent analysis was conducted only on the HbO signal.

\section{Results}

Preliminary analysis-All participants answered correctly the questions regarding the content of each of the two stories. None reported having ever heard the "un-known" story Martine and the Witch and all of them were quite familiar with the "known" story Snow White. Finally, EEG monitoring revealed no signs of drowsiness in any condition.

Using the international 10-20 system [25], six regions were delimited for all subjects: left frontal-temporal (F7 and T3), left medial-temporal (T3 and T5) left temporal-parietal (T5 and P3), as well as the right homologous areas (see Figure 1(a)). For each of these regions, oxyhemoglobin (HbO) and deoxyhemoglobin (HbR) changes during task periods were examined and the region of interest (ROI) was selected using the maximal amplitude and duration of $\mathrm{HbO}$ changes. Each subject's ROIs were then fitted on a segmented Atlas 116 template [26].

In response to French, the regions of activation found in all subjects included the superior temporal gyrus, the middle temporal gyrus and the angular gyrus (see Figure 1(b) for a representation of the activated areas). Subsequent analyses were conducted on these individual ROIs.

Figures 2 and $\mathbf{3}$ show the hemodynamic changes recorded in response to the known and the unknown stories respectively in both languages for subject GT whose results are representative of the group. For the French condition, $\mathrm{HbO}$ concentration increased at the beginning of the story segment in the left hemisphere (blue lines), while in the right hemisphere only a small increase was observed (red lines). A maximal peak was recorded around $6 \mathrm{~s}$ after the beginning of the stimulus in both hemispheres and for both stories. However, the amplitude of the change in the 
signal induced by the story in French was higher and lasted longer in the left hemisphere (until $16 \mathrm{~s}$ after the beginning of the stimulus compared to $10 \mathrm{~s}$ in the right hemisphere). This effect was observed for both stories (known and unknown stories). In contrast, the change in the hemodynamic signal recorded in response to the story in Arabic showed a decrease starting at $9 \mathrm{~s}$ after the beginning of the stimulus in both hemispheres and for both stories.

In order to assess the hemispheric lateralization pattern, a comparison by subtraction was performed. For each of the four conditions, the hemodynamic signal induced in the right hemisphere was subtracted from the hemodynamic signal observed in the left hemisphere. The area under the curve (AUC) of the subtraction for $\mathrm{HbO}$ concentration (between 0 and 25 seconds) was then used as a measure of lateralization. According to this calculation, a positive HbO AUC would suggest left hemisphere dominance while a negative $\mathrm{HbO}$ AUC would suggest right hemisphere dominance. Figures 2(e), 2(f) and 3(e), 3(f) display the results of the subtraction for subject GT. The group mean (four subjects) and standard error of AUC for each condition are plotted in Figure 4.

Effects of language and story familiarity were further assessed using a two-way repeated measure ANOVA
(French/Arabic language for known/unknown story), in which the dependent measure was the AUC resulting from the subtraction (left AUC minus right AUC). As expected, results revealed a significant main effect for language $[F(1,3)=10.33, p<0.05]$ with a large effect size (eta square $=0.78$ ). The story familiarity (known vs. unknown) was not significant $[F(1,3)=0.12$, $p=0.75$, eta square $=0.04]$ neither was the interaction language by familiarity effect $[F(1,3)=0.51, p=0.84$, eta square $=$ 0.02].

\section{Discussion}

Our findings indicate that the hemodynamic signal changes associated to French stories are greater in the left than the right temporal regions, suggesting a left hemisphere dominance for receptive language in French-speaking participants. In contrast, Arabic stories (i.e. unknown language) did not induce specific hemispheric patterns. Consistent with previous reports $[5,10]$ using similar listening tasks in fMRI or PET, the left superior temporal gyrus and left middle temporal gyrus, were particularly activated. Despite the limited sample size of our study, a very large effect size for language was found, further supporting the efficacy of optical imaging in assessing receptive language lateralization processes.
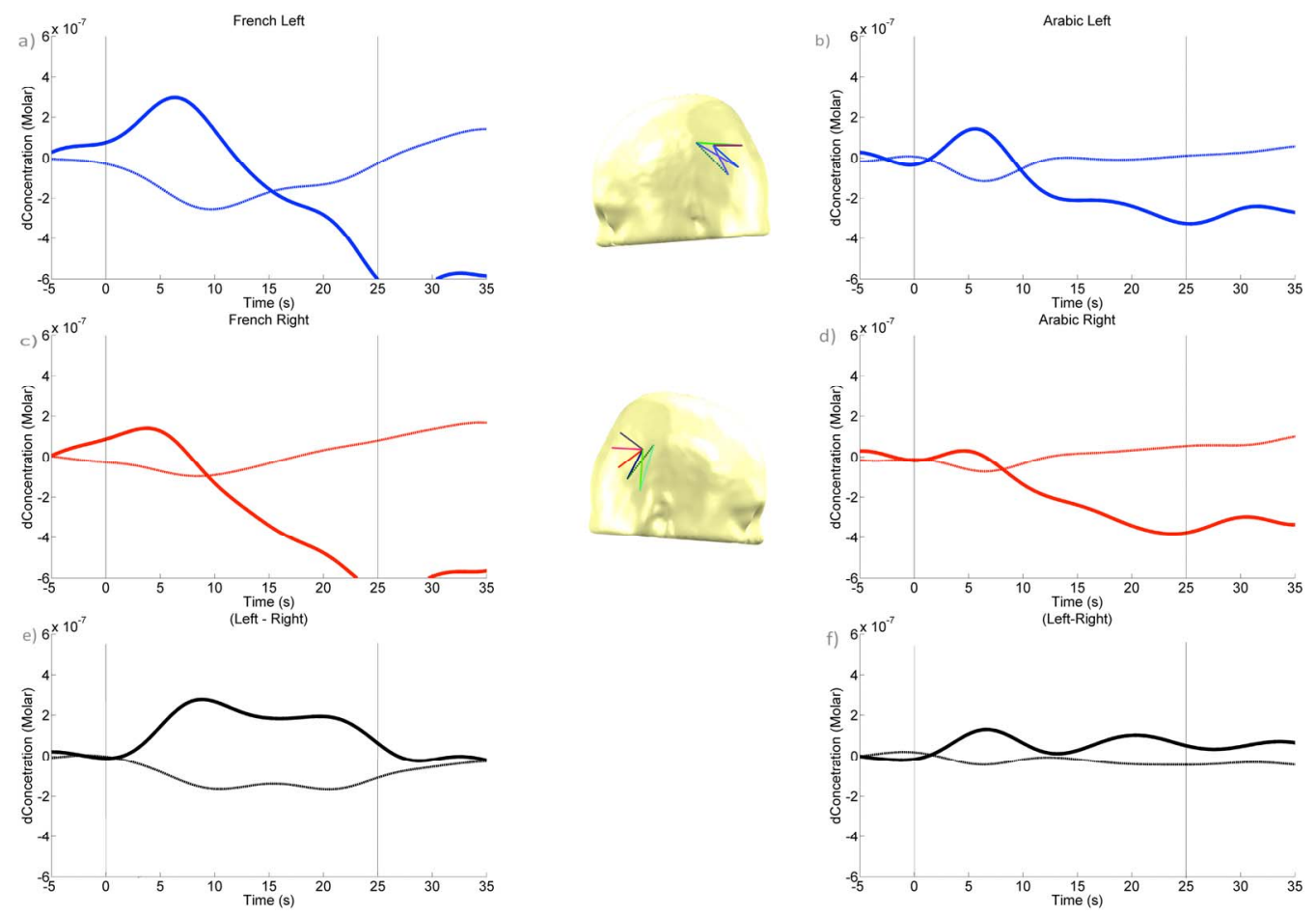

Figure 2. Hemodynamic responses to the known story for subject GT. The y-axis indicates relative changes in concentration (micromolar) for $\mathrm{HbO}$ (continuous line) and HbR (dotted line) during presentation of the story segments (20 s). Hemodynamic changes during stories in French in the left and right hemispheres are represented respectively in (a) and (c), whereas hemodynamic changes during stories in Arabic in the left and right hemispheres are represented in (b) and (d). Hemispheric subtractions of the hemodynamic signal (left minus right) are illustrated for the story told in French (e) and in Arabic (f). 

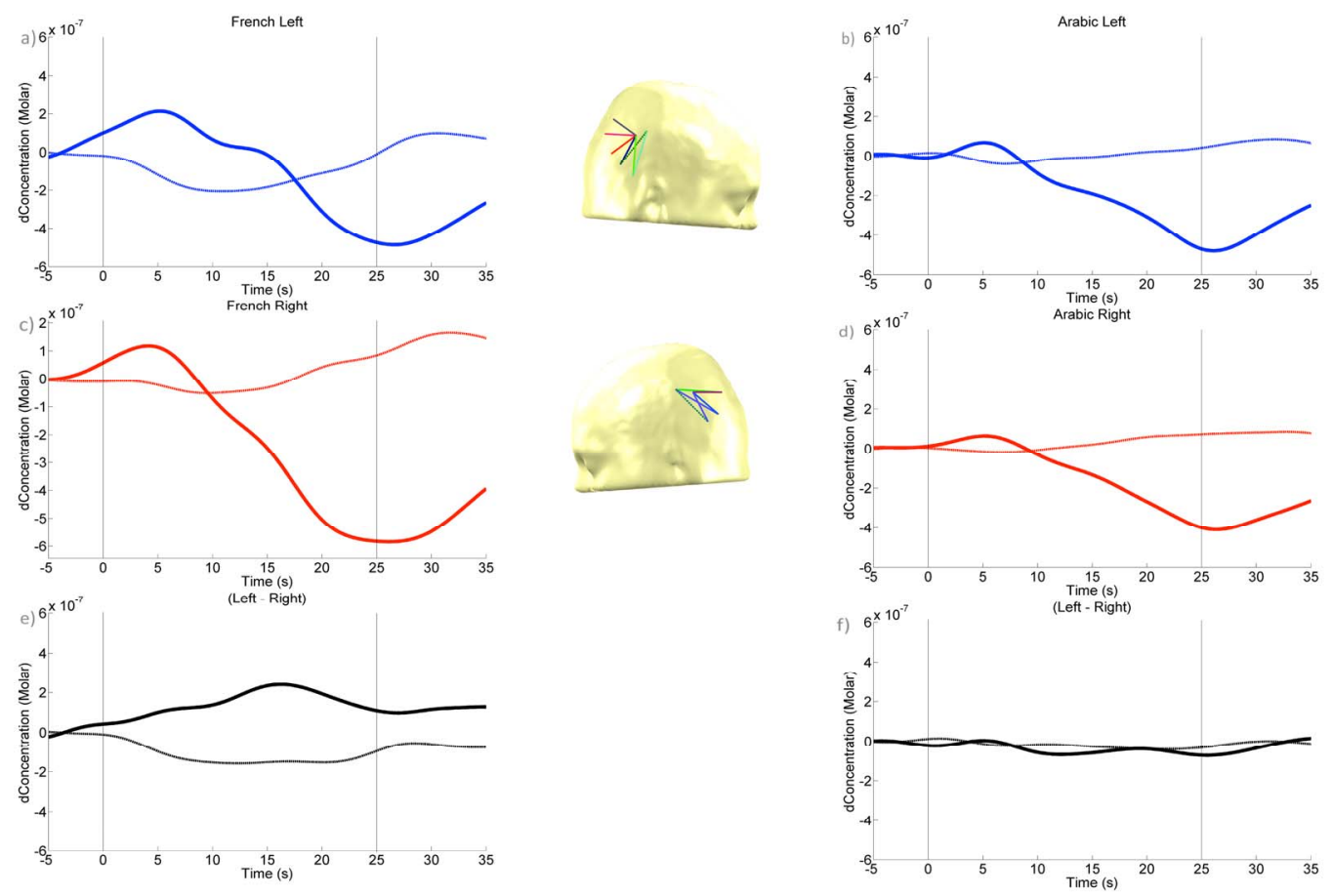

Figure 3. Hemodynamic responses to the unknown story for the same participant (GT). The y-axis indicates relative changes in concentration (micromolar) for $\mathrm{HbO}$ (continuous line) and HbR (dotted line) during the presentation of the story segments (20 s). Hemodynamic changes during the story in French in the left and right hemispheres are represented respectively in (a) and (c), whereas hemodynamic changes during the story in Arabic in the left and right hemisphere are represented in (b) and (d).

The findings of the present study also provide a new approach to calculate language hemispheric lateralization. Previous studies have mainly used the Laterality Index (LI) formula $(\mathrm{L}+\mathrm{R} / \mathrm{L}-\mathrm{R})$ where $\mathrm{L}$ is the maximal peak found in the left hemisphere and $\mathrm{R}$ is the maximal peak found in the right hemisphere. The usual LI value goes from -1 (right hemisphere dominance) to +1 (left hemisphere dominance). However, the interpretation of the laterality index depends on the use of subjective parameters such as number of voxels used in fMRI, the maximal peak, or the selected timing

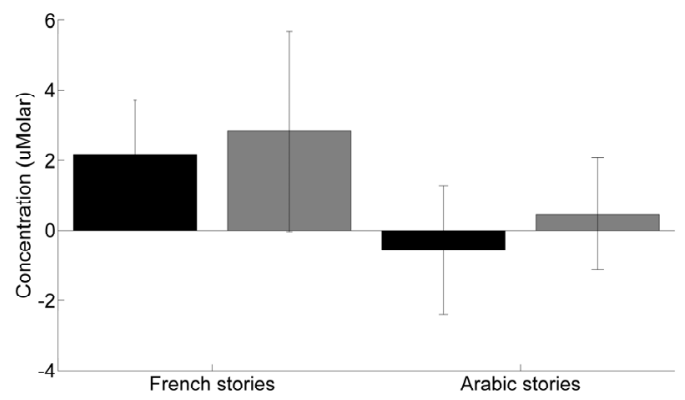

Figure 4. Average $(n=4)$ of the hemispheric lateralization.

(Area Under the Curve HbO Left - AUC HbO right) in response to the known story (black bars) and the unknown story (gray bars) in both languages. The $\mathrm{y}$-axis indicates average changes in concentration (micromolar) for $\mathrm{HbO}$ Error bars indicate the standard errors. Positive HbO AUC indicates left hemisphere specialization while negative HbO AUC suggests right hemisphere specialization.
[27]. Moreover, its computation is only possible with positive or absolute values, which limits its use with blood-oxygen level dependent measures (BOLD) as in optical imaging and fMRI. In the present study, we suggest that subtracting the hemodynamic signal of the hemispheric regions (Area under the Curve HBO Left-AUC HBO Right) provides a more objective measure of hemispheric lateralization. This new method may be more suitable for optical imaging as it takes into account the decrease in $\mathrm{HbO}$ concentration. The use of the subtracted AUC values as a measure of lateralization may thus provide a more confident index because it better reflects the variation of $\mathrm{HbO}$ concentration all along the story segments. Further validation of this new method for measuring language lateralization is needed.

Finally, this study also aimed to determine whether the presentation of a well known story would affect the activation patterns observed in specific brain regions involved in memory of imagery processes. Our results suggest that participants responded similarly to the known and unknown stories, regardless of the episodic memory retrieval expected while listening to the known story. This result confirms the robustness of our paradigm in assessing language rather than memory processes.

In summary, our findings confirm the use of optical imaging as a reliable and simple tool to study receptive language lateralization patterns in adults using story comprehension paradigms. Taking into account the numer- 
ous advantages of NIRS, these results are encouraging for further investigation of language development in children as well as for receptive language lateralization patterns in special populations such as patients with mental retardation and/or epileptic patients. This is of special importance, considering the fact that these populations often show restricted capabilities making other imaging methods inappropriate to study language lateralization which, in cases of intractable epilepsy, constitute a necessary part of the pre-surgical investigation.

\section{REFERENCES}

[1] B. Abou-Khalil and B. L. Schlaggar, "Is It Time to Replace the Wada Test?” Neurology, Vol. 59, No. 2, 2002, pp. 160-161.

[2] A. Gallagher, M. Thériault, E. Maclin, K. Low, G. Gratton, M. Fabiani, L. Gagnon, K. Valois, I. Rouleau, H.C. Sauerwein, L. Carmant, D.K. Nguyen, A. Lortie, F. Lepore, R. Béland and M. Lassonde, "Near-Infrared Spectroscopy as an Alternative to the Wada Test for Language Mapping in Children, Adults and Special Populations," Epileptic Disorders : International Epilepsy Journal with Videotape, Vol. 9, 2007, pp. 241-255.

[3] N. F. Watson, C. Dodrill, D. Farrell, M. D. Holmes, and J. W. Miller, "Determination of Language Dominance with Near-Infrared Spectroscopy: Comparison with the Intracarotid Amobarbital Procedure,” Seizure: The Journal of the British Epilepsy Association, Vol. 13, 2004, pp. 399-402.

[4] A. Gallagher, D. Bastien, I. Pelletier, P. Vannasing, A. D. Legatt, S. L. Moshé, R. Jehle, L. Carmant, F. Lepore, R. Béland and M. Lassonde, “A Noninvasive, Presurgical Expressive and Receptive Language Investigation in a 9-Year-Old Epileptic Boy Using Near-Infrared Spectroscopy,” Epilepsy \& Behavior, Vol. 12, No. 2, 2008, pp. 340-346. doi:10.1016/j.yebeh.2007.10.008

[5] B. M. Mazoyer, N. Tzourio, V. Frak, A. Syrota, N. Murayama, O. Levrier, G. Salamon, S. Dehaene, L. Cohen and J. Mehler, "The Cortical Representation of Speech," Journal of Cognitive Neuroscience, Vol. 5, No. 4, 1993, pp. 467-479. doi:10.1162/jocn.1993.5.4.467

[6] C. Narain, "Defining a Left-lateralized Response Specific to Intelligible Speech Using fMRI,” Cerebral Cortex, Vol. 13, No. 12, 2003, pp. 1362-1368. doi:10.1093/cercor/bhg083

[7] C. Tse, C. Lee, J. Sullivan, S. M. Garnsey, G. S. Dell, M. Fabiani and G. Gratton, "Imaging Cortical Dynamics of Language Processing with the Event-Related Optical Signal," Proceedings of the National Academy of Sciences of the United States of America, Vol. 104, No. 43, 2007, pp. 17157-17162. doi:10.1073/pnas.0707901104

[8] N. Ikuta, M. Sugiura, Y. Sassa, J. Watanabe, Y. Akitsuki, K. Iwata, N. Miura, H. Okamoto, Y. Watanabe, S. Sato, K. Horie, Y. Matsue and R. Kawashima, "Brain Activation during the Course of Sentence Comprehension,” Brain and Language, Vol. 97, No. 2, 2006, pp. 154-161. doi:10.1016/j.bandl.2005.10.006
[9] G. R. Kuperberg, P. K. McGuire, E. T. Bullmore, M. J. Brammer, S. Rabe-Hesketh, I. C. Wright, D. J. Lythgoe, S. C. Williams and A. S. David, "Common and Distinct Neural Substrates for Pragmatic, Semantic, and Syntactic Processing of Spoken Sentences: An Fmri Study," Journal of Cognitive Neuroscience, Vol. 12, No. 2, 2000, pp. 321-341. doi:10.1162/089892900562138

[10] M. J. Schlosser, N. Aoyagi, R. K. Fulbright, J. C. Gore and G. McCarthy, "Functional MRI Studies of Auditory Comprehension.," Human Brain Mapping, Vol. 6, No. 1, 1998, pp. 1-13. doi:10.1002/(SICI)1097-0193(1998)6:1<1::AID-HBM1>3.0.C $\underline{\mathrm{O} ; 2-7}$

[11] B. Mazoyer, L. Zago, E. Mellet, S. Bricogne, O. Etard, O. Houdé, F. Crivello, M. Joliot, L. Petit and N. TzourioMazoyer, "Cortical Networks for Working Memory and Executive Functions Sustain the Conscious Resting State in Man,” Brain Research Bulletin, Vol. 54, 2001, pp. $287-$ 298. doi:10.1016/S0361-9230(00)00437-8

[12] R. A. Mar, "The Neuropsychology of Narrative: Story Comprehension, Story Production and Their Interrelation," Neuropsychologia, Vol. 42, No. 10, 2004, pp. 1414-1434. doi:10.1016/j.neuropsychologia.2003.12.016

[13] S. Gardini, C. Cornoldi, R. De Beni and A. Venneri, "Left Mediotemporal Structures Mediate the Retrieval of Episodic Autobiographical Mental Images,” NeuroImage, Vol. 30, No. 2, 2006, pp. 645-655. doi:10.1016/j.neuroimage.2005.10.012

[14] I. Pelletier, H. C. Sauerwein, F. Lepore, D. Saint-Amour and M. Lassonde, "Non-Invasive Alternatives to the Wada Test in the Presurgical Evaluation of Language and Memory Functions in Epilepsy Patients," Epileptic Disorders: International Epilepsy Journal With Videotape, Vol. 9, 2007, pp. 111-126.

[15] A. Villringer, J. Plank, C. Hock, L. Schleinkofer and U. Dirnagl, “Near Infrared Spectroscopy (Nirs): A New Tool to Study Hemodynamic Changes during Activation of Brain Function in Adults," Neuroscience Letters, Vol. 154, 1993, pp. 101-104. doi:10.1016/0304-3940(93)90181-J

[16] A. Villringer and B. Chance, "Non-Invasive Optical Spectroscopy and Imaging of Human Brain Function. Trends in Neuroscience,” Vol. 20, No. 10, October 1997, pp. 435-442. doi:10.1016/S0166-2236(97)01132-6

[17] E. Watanabe, Y. Yamashita, A. Maki, Y. Ito and H. Koizumi, "Non-Invasive Functional Mapping with MultiChannel Near Infrared Spectroscopic Topography in Humans," Neuroscience Letters, Vol. 205, 1996, pp. 4144. doi:10.1016/0304-3940(96)12376-4

[18] M. Ohnishi, N. Kusakawa, S. Masaki, K. Honda, N. Hayashi, Y. Shimada, I. Fujimoto and K. Hirao, "Measurement of Hemodynamics of Auditory Cortex Using Magnetoencephalography and Near Infrared Spectros-Copy," Acta Oto-Laryngologica, Vol. 117, 1997, pp. 129-131. doi:10.3109/00016489709126161

[19] E. Watanabe, a. Maki, F. Kawaguchi, K. Takashiro, Y. Yamashita, H. Koizumi and Y. Mayanagi, "Non-Invasive Assessment of Language Dominance with Near-Infrared Spectroscopic Mapping,” Neuroscience letters, Vol. 256, 
No. 1, 1998, pp. 49-52. doi:10.1016/S0304-3940(98)00754-X

[20] R. P. Kennan, D. Kim, A. Maki, H. Koizumi, and R. T. Constable, "Non-Invasive Assessment of Language LaterAlization by Transcranial Near Infrared Optical Topography and Functional MRI,” Human Brain Mapping, Vol. 16, 2002, pp. 183-189. doi:10.1002/hbm.10039

[21] R. Oldfield, "The Assessment and Analysis of Handedness: The Edinburgh Inventory,” Neuropsychologia, Vol. 9, No. 1, March 1971, pp. 97-113. doi:10.1016/0028-3932(71)90067-4

[22] A. C. Evans, D. L. Collins and B. Milner, "An MRIBased Stereotactic Atlas from 250 Young Normal Subjects," Society for Neuroscience Abstracts, Vol. 18, No. 179, 1992, p. 408.

[23] A. Duncan, J. H. Meek, M. Clemence, C. E. Elwell, P. Fallon, L. Tyszczuk and D. T. Delpy, "Measurement of Cranial Optical Path Length as a Function of Age Using Phase Resolved Near Infrared Spectroscopy,” Pediatric Research, Vol. 39, No. 5, May 1996, pp. 889-894. doi:10.1203/00006450-199605000-00025
[24] G. Strangman, "Factors Affecting the Accuracy of NearInfrared Spectroscopy Concentration Calculations for Focal Changes in Oxygenation Parameters,” NeuroImage, Vol. 18, No. 4, 2003, pp. 865-879. doi:10.1016/S1053-8119(03)00021-1

[25] R. Homan, J. Herman and P. Purdy, "Cerebral Location of International 10-20 System Electrod Placement,” Electroencephalography and Clinical Neurophysiology, Vol. 66, No. 4, 1987, pp. 376-382. doi:10.1016/0013-4694(87)90206-9

[26] D. L. Collins, A. Zijdenbos, V. Kollokian, J. G. Sled, N. J. Kabani, C. J. Holmes and A. C. Evans, "Design and Construction of a Realistic Digital Brain Phantom,” IEEE Transaction on Medical Imaging, Vol. 17, No. 3, June 1998, pp. 463-468. doi:10.1109/42.712135

[27] M. L. Seghier, "Laterality Index in Functional MRI: Methodological Issues,” Magnetic Resonance Imaging, Vol. 26, No. 5, 2008, pp. 594-560. doi:10.1016/j.mri.2007.10.010 